\title{
Urinary tract infections in children after renal transplantation
}

\author{
Ulrike John • Markus J. Kemper
}

Received: 4 June 2007 /Revised: 2 October 2007 / Accepted: 23 October 2007 / Published online: 16 January 2008

(C) IPNA 2007

\begin{abstract}
Urinary tract infections (UTI) after pediatric kidney transplantation (KTX) are an important clinical problem and occur in $15-33 \%$ of patients. Febrile UTI, whether occurring in the transplanted kidney or the native kidney, should be differentiated from afebrile UTI. The latter may cause significant morbidity and is usually associated with acute graft dysfunction. Risk factors for (febrile) UTI include anatomical, functional, and demographic factors as well as baseline immunosuppression and foreign material, such as catheters and stents. Meticulous surveillance, diagnosis, and treatment of UTI is important to minimize acute morbidity and compromise of long-term graft function. In febrile UTI, parenteral antibiotics are usually indicated, although controlled data are not available. As most data concerning UTI have been accumulated retrospectively, future prospective studies have to be performed to clarify pathogenetic mechanisms and risk factors, improve prophylaxis and treatment, and ultimately optimize long-term renal graft survival.
\end{abstract}

Keywords Kidney transplantation - Urinary tract infection . Transplant pyelonephritis · Immunosuppression .

Vesicoureteric reflux $\cdot$ Neurogenic bladder

U. John

University Children's Hospitals,

Kochstr. 2,

07745 Jena, Germany

M. J. Kemper $(\square)$

Klink für Kinder-und Jugendmedizin,

Martinistr. 52,

20246 Hamburg, Germany

e-mail: kemper@uke.uni-hamburg.de

\section{Introduction}

Kidney transplantation (KTX) is now considered the treatment of choice for end-stage renal disease (ESRD) in children [1]. Patient survival is approaching $100 \%$, and graft survival has improved significantly in recent years, mainly due to improved immunosuppressive strategies [2]. The main problem, however, chronic allograft nephropathy (CAN), remains unresolved. In CAN, specific immunological but also nonimmunological risk factors, such as hypertension and urinary tract infections (UTI), seem to play a role [3, 4], so that the ultimate solution to the problem will rely on several approaches.

Febrile UTI play a crucial role in progression of chronic kidney disease even before KTX and is often associated with anatomical urogenital abnormalities. Also, after KTX, UTI may lead to kidney damage, negatively affecting longterm graft survival by scarring and interstitial injury [5-7], although precise data for the pediatric population are scarce [8-10]. UTI management in such patients undoubtedly is more complex compared with UTI in otherwise healthy children, so that extrapolation of data is not always possible. Furthermore hardly any current diagnostic and/or treatment practices in such patients are evidence-based.

The aim of this teaching article is to summarize the clinical relevance and details of UTI after renal transplantation. Our focus is mainly on febrile UTI (fUTI), although afebrile UTI is also discussed. We discuss specific pathogenetic risk factors as well as options for treatment and prophylaxis of this frequent complication after KTX.

\section{Diagnosis of UTI after KTX}

In general, UTI diagnosis in healthy children and those with urinary tract malformations or after KTX should follow the 
same principles. However, such patients after renal transplantation are more complex, as they are immunocompromised, often have anatomical urinary tract abnormalities, and maintenance of organ function is pivotal.

UTI diagnosis is confirmed with growth of $10^{5}$ colonies of bacteria in a collected sample. As in the case in healthy children, the method of urine collection is important in transplant recipients. Bagged specimens are unreliable due to the high degree of contamination, and midstream and catheterized urine samples are preferred in infants and older children, depending on clinical status. Suprapubic tap remains the most accurate method with the least contamination but is not used much in clinical transplantation [11, 12]. It is the only method that can exclude bacterial or fungal contamination with certainty, however. An alternative, though invasive method, of obtaining bladder urine with minimal contamination is by means of catheterization. Introduction of infection is feasible by this method, trauma of the urethra may occur, and psychological stress for patient (and doctor) may be high. The method may be superior in the ill child, however, when urinalysis is required quickly, especially when bladder volume is low [11].

Urinary abnormalities in UTI include leukocyturia, hematuria, and positive leukocyte esterase, often identified by dipstick testing. The presence of white blood cells (WBC) in urine is not limited to UTI but may occur in other conditions, such as acute rejection, BK nephropathy, and presence of foreign material, such as a stent, in the urinary tract. Data on the diagnostic impact of these tests in KTX are not available.

In fUTI after KTX (transplant pyelonephritis), elevation of inflammatory markers are typical, such as leucocytosis, erythrocyte sedimentation rate, and C-reactive protein (CRP). These parameters are not specific for UTI, but they may be important to distinguish between transplant pyelonephritis and rejection episodes [13], as renal dysfunction is a typical feature for both. They are also important in follow-up, as they are tools for monitoring response to antibiotic treatment [14].

There are few data concerning the optimal diagnostic imaging during fUTI after KTX. Ultrasound is a noninvasive tool and may demonstrate hydronephrosis or swelling of the transplanted kidney. Perfusion deficits may be visualized by color-Doppler ultrasound, especially in the power mode (Fig. 1). Dimercaptosuccinate acid (DMSA) scintigraphy is regarded as the gold standard in the diagnosis of acute pyelonephritis as well as in the documentation of residual scarring. This tool has been employed only occasionally in UTI after renal transplantation (Fig. 2). Long-term data suggest that fUTI may lead to focal defects on DMSA scanning [5, 15], but also other events, such as vascular complications and renal biopsies, may do so, which has to be considered in the interpretation of this diagnostic tool [16]. However, at present, no clear recommendations can be made as to the use of DMSA

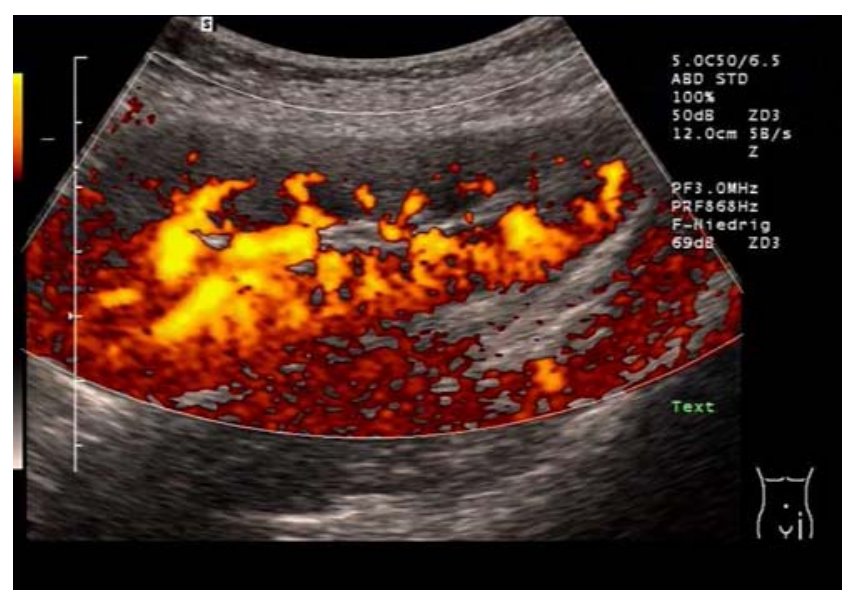

Fig. 1 Longitudinal power Doppler ultrasonogram of a patient with transplant pyelonephritis showing vascular hypoperfusion in the cortex

scanning as a diagnostic tool in transplant pyelonephritis, neither in the acute phase, nor in follow-up.

\section{Classification of UTI after KTX}

Most importantly, afebrile UTI must be distinguished from fUTI. Afebrile UTI may also affect the native kidneys, which is a diagnostic dilemma. Patients with afebrile UTI after KTX may be symptomatic (dysuria, frequency, etc.) or may be completely asymptomatic, raising the question of whether treatment is indicated. Furthermore, UTI, both febrile and afebrile, may show recurrences. It should be noted that immunosuppression, especially with steroids, might alter the temperature response to infections by suppressing cytokine production.

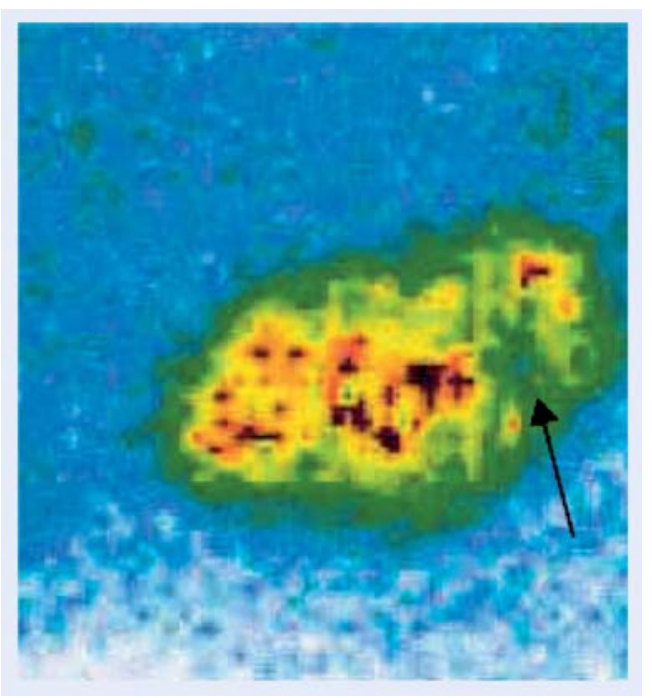

Fig. $299 \mathrm{mTc}$ DMSA scan of a patient with acute transplant pyelonephritis showing an uptake defect in the lower pole (arrow) 


\section{Clinical features}

Fever and renal dysfunction are the typical features of fUTI after KTX. In some patients, symptoms and signs of urosepsis may develop. The acute deterioration of renal function during UTI is a well-known feature [6, 17] which, together with the significant inflammatory parenchymal response, emphasizes the potential risk of tissue damage in the transplanted kidney in acute and long-term follow-up, despite normalization of serum creatinine values after UTI. Acute rejection episodes may be triggered by fUTI [18], and development of an intrarenal abscess in the graft following UTI has also been described [19].

\section{Prevalence}

A high prevalence of fUTI in children after renal transplantation has been demonstrated by several retrospective studies. They are not limited to the immediate posttransplant period but also occur later, especially in girls [14]. In pediatric studies, the prevalence ranged between $15 \%$ and $33 \%[6,9,10,17]$. Higher prevalence of up to $61 \%$ has been demonstrated in adults; however, in some studies, entry criteria were less strict, such as inclusion of patients with (asymptomatic) afebrile bacteriuria [20, 21]. Recently, Pelle et al. demonstrated a prevalence of UTI of $75.1 \% ; 18.7 \%$ of these patients developed transplant pyelonephritis [22]. Other studies have found a higher frequency in children; for instance, admissions for UTIs in pediatric patients after renal transplants in the United States averaged nearly 150 per 1,000 patient years at risk, compared with 67 among adults (editorial comment following [23]).

\section{Epidemiology}

Although Escherichia coli remained the most frequently isolated microorganism, as in other studies [8, 24, 25], it was isolated less frequently than in the general pediatric population, where it is found in up to $80 \%$ of UTIs [26]. This may be due to the underlying immunosuppression and colonization. Also, some patients receive antibiotic prophylaxis or treatment, which may have an impact on colonization. Surveillance urinary cultures may help, especially for patients with recurrent UTI and those with abnormal lower urinary tract and neurogenic bladder; not infrequently, bacteria with multiple antibiotic resistances, such as Pseudomonas sp., can be detected. Table 1 demonstrates epidemiology of fUTI in published series as well as in control populations.

\section{Risk factors}

Several risk factors for UTI have been elucidated by studies in adults and children. Knowledge of these risk factors is one key for prevention.

1. Anatomical factors

Underlying urological abnormalities have to be regarded as risk factors for UTI even before transplantation, such as hydronephrosis, vesicoureteric reflux (VUR), and/or neurogenic bladder, often in combination. Surgery of the urinary tract before listing for transplantation is often necessary and should decrease the risk of infections, although in our retrospective study, operated children per se had more UTIs, possibly because patients represent a specific population at risk, even after surgery [14]. Patients with urinary tract malformations compromise about a third of children with end-stage renal failure and include heterogeneous disorders such as posterior urethral valves, prune-belly syndrome, spina bifida, or urogenital sinus abnormalities, which may lead to lower urinary tract dysfunction [27-30]. In our study, structural disorders and neurogenic bladder were present in $33 \%$ of all patients with fUTI.

Especially structural abnormalities of the bladder anatomy and neurogenic bladder have been regarded as specific risk factor that needs to be distinguished from behavioral voiding problems (voiding dysfunction). Neurogenic bladder leads to a higher incidence of UTI, with associated morbidity and poorer graft function [31]. The presence of focal scars was associated with either raised intravesical pressures or recurrent UTIs [5]. Although no controlled data are available that bladder augmentation procedures prevent or decrease the rate of UTI after transplantation, it seems mandatory that evaluation and treatment of neurogenic bladder is very important and should be performed before renal transplantation, requiring specific follow-up after KTX.

2. Functional factors

In addition to abnormal bladder anatomy and neurogenic bladder, voiding dysfunction is present in many patients and may also increase the risk of UTI in analogy to the nontransplant population [32]. On the other hand, a recent retrospective study from Herthelius et al. [33, 34] suggested no increased frequency of UTI after KTX in children with bladder dysfunction. However, the study did not analyze the effect of interventions in the patients with voiding dysfunction.

3. Vesicoureteric reflux (VUR)

Among the anatomical factors, the pathogenetic role of VUR has been discussed as an especially important risk factor for UTI after renal transplantation. A clear dis- 


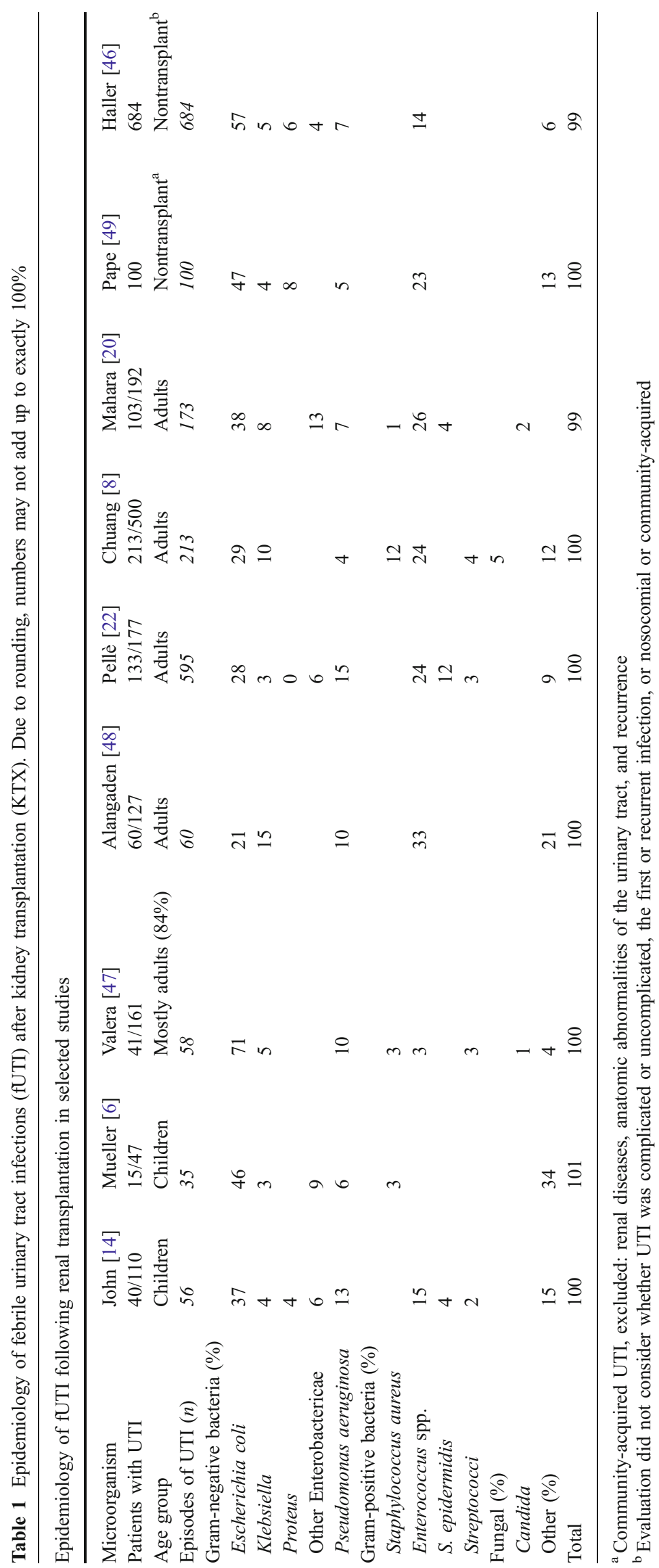


tinction has to be made, however. First, VUR is present in many patients as an underlying diagnosis, and UTI could affect the native and the transplanted kidney (or both). The differential diagnosis between these entities is almost impossible without sophisticated imaging studies. Some authors recommend native nephrectomy of kidneys with VUR to decrease the risk of native-kidney UTI after KTX [35]. However, no controlled studies are available. Also, pretransplant antireflux surgery did not reduce the risk for fUTI after KTX in a recent study of Basiri et al., who compared 12 children with VUR who underwent reimplantation to 17 children with VUR who did not [36]. The lack of difference may be due to dysfunctional voiding that is also often present in children with VUR and that may be an independent risk factor for UTI, of both native and transplanted kidney.

Second, VUR into the transplanted kidney in a previously normal urinary tract may develop secondary to transplant surgery, thereby increasing the risk for transplant pyelonephritis. Dunn et al. [10] found an increased incidence of pyelonephritis in pediatric renal transplant patients who had VUR. Ranchin et al. tested 55 of 84 transplanted children during a period of 5 years [9] and demonstrated a 58\% prevalence of VUR. The rate of UTI was $60 \%$ and was similar in patients with or without VUR in the overall analysis. However, after excluding UTI related to catheterization, the rate of transplant pyelonephritis was higher in patients with VUR $(p<0.02)$. Whether strict antireflux surgery can reduce the risk of fUTI after renal transplantation has not been studied so far. Correction of vesicoureteric reflux into the kidney graft has been shown to reduce the incidence of UTI in a small series but was associated with obstructive complications [17], which may be high, especially in the cohort with associated abnormal bladder anatomy.

4. Stents and other manipulations

Foreign material, such as stents, urinary catheters, and suture material, can cause UTI, as it is frequently colonized by bacteria (and fungi). In the study by Kamath et al., the odds ratio for UTI for ureteric stents was 4.6 [37]. Therefore, these devices should only be used for a short time [20] or avoided if possible. Manipulation with or insertion of such foreign material should therefore be performed with caution and possibly with antibiotic prophylaxis, especially in the initial posttransplant period, when high-dose immunosuppression increases the risk even further.

5. Gender

The rate of UTI in adult females has been reported to be twice as high as in male renal transplant recipients [8, $20,38]$, and we were able to confirm this finding for children in our retrospective multicenter analysis [14].
Anatomical reasons (e.g. shortness of urethra) may be relevant in children and adults, and in female adolescents, sexual activities have to be taken into account (and asked about), as well as predisposing immunological factors in females [39].

6. Immunosuppression

Immunosuppression has an impact on defense mechanisms so that a theoretical impact on the incidence of UTI should be present. A recent study indicated an increased risk for patients on a mycophenolic-based regimen, with an odds ratio of 1.9 [37], but not for other immunosuppressants, especially induction treatment with anti-CD3. Therefore, patients on high-dose immunosuppression should be regarded as at risk for bacterial and viral infections, including UTI. An accumulation of risk factors (e.g. unnecessary catheterization or manipulation at the urinary tract, should be avoided or at least limited. Future treatment studies should, in detail, also look at the impact of specific immunosuppressants on infection, including UTI.

In our opinion, it should be emphasized that a combination of risk factors occurs in many patients and that it is unlikely that a single factor is responsible for the development of febrile or afebrile UTI.

Do urinary tract infections affect long-term outcome?

Several reports indicate that despite acute graft dysfunction during fUTI, long-term renal function was not different between patients with and without infection [14, 40, 41]. Other reports show a less optimistic picture in patients with UTI, not only in adults [22], but especially in children $[9,34]$, and even more for those with recurrent UTIs [34]. In this respect, especially the retrospective cohort study of Abbott et al. in nearly 29,000 adult renal transplant patients of the United States Renal Data System (USRDS) database is of interest, because late UTIs were independently associated with the risk of subsequent graft loss and even death [38]. It remains difficult, however, to properly assess the risk in these uncontrolled and partially selective series, especially in view of an imperfect endpoint (i.e. serum creatinine). It is also of special note that also in asymptomatic (afebrile) UTI, serum creatinine increases have been reported [24].

More studies into the pathophysiology of UTI after KTX are necessary in this respect. Serotypes and adherence factors of bacteria such as Escherichia coli may have a strong impact on acute and long-term graft dysfunction, as suggested by a recent study from Rice et al. [42]. The intensive inflammatory response indicated by CRP or other markers, such as procalcitonin response, may be related to the 
degree of tissue destruction leading to fibrosis [43]. The concurrent treatment with steroids with their anti-inflammatory properties may possibly even be a protective factor [44]. All these issues need to be addressed to clarify the issue of longterm endangerment. Last but not least, long-term clinical variables need to be defined and collected.

Treatment of UTI after renal transplantation

Aggressive and specific treatment of fUTI is mandatory. In our opinion, in fUTI, parenteral antibiotics should be preferred, at least initially, to achieve fast tissue saturation. The optimal treatment duration has not been studied, but we favor 14 days in transplant pyelonephritis. In accordance with Benador et al., we sometimes continue with oral treatment when the clinical situation has improved [45]. As Enterococcus and Pseudomonas spp. are more frequent, we currently use a combination of ceftazidime and ampicillin to cover for E. coli, Pseudomonas, and Enterococcus. Others have recommended ampicillin and gentamicin for the same reason [46]. However, nephrotoxicity of the latter is a concern. Oral fluoroquinolone medications such as ciprofloxacin is another alternative in this situation. Fungal UTIs may occur and require specific treatment; in our center, antifungal prophylaxis is given during highdose antibiotic treatment. Treatment recommendation for other centers may be different, however. Monitoring of blood levels is important during acute UTI, as antibiotic treatment may interfere with resorption. Steroid dose must be increased sometimes during fUTI, especially early after renal transplantation, to avoid symptoms of adrenal insufficiency.

Symptomatic afebrile UTI may be treated with oral antibiotics unless specific risk factors are present (renal dysfunction etc.) [11]. Again, treatment should be specific and oral cephalosporin may be the first choice, especially if the patient was on antibiotic prophylaxis. Whether asymptomatic UTIs have to be treated remains controversial and is often an individual decision. In patients with abnormal bladder anatomy and catheterization, such as in spina bifida, colonization is frequent, and symptoms such as dysuria may be absent. There is no evidence and no consensus as to whether in these patients bacterial colonization needs treatment, including bladder washing with antibiotics. In our centers, we currently only treat symptomatic patients with abnormal bladder anatomy and bacterial colonization and do not use antibiotic bladder irrigation.

Practical consequences for the clinician

Although UTIs cannot be prevented completely, preventative measures may be able to reduce the incidence and lead to earlier and more effective treatment. Recurrences of fUTI should be prevented, if at all possible.

1. Antibiotic prophylaxis

There are no systematic studies relating to use of prophylactic antibiotics after renal transplantation. They may be useful in the view of the high prevalence of UTI, however, and most pediatric transplant centers prescribe prophylactic antibiotics for the first 3-6 months posttransplantation, or longer in refluxive patients [31, 41], but sometimes also for Pneumocystis carinii prophylaxis. However, not all studies confirmed a beneficial effect and even demonstrated a high bacterial resistance rate [24]. If prophylactic antibiotics are used, it seems that they should be administered for prolonged periods, as fUTI may occur late, especially in girls.

2. Bladder dysfunction

As bladder dysfunction contributes to the risk of UTI, exact diagnosis (and treatment) before transplantation and surveillance is important. Simple methodology (micturition protocols, uroflowmetry, and bladder ultrasound) for this evaluation exists and needs to be used. Sometime, however, it is not possible to evaluate bladder function before transplantation, e.g. in the anuric patient who never had a voiding history. Patients with structural and functional bladder abnormalities require regular urodynamic monitoring and treatment: pharmacologic, behavioral, or surgical. This includes frequent voiding, treatment of constipation, and sometimes intermittent catheterization. Correction of structural anomalies and optimization of storage and emptying functions of the bladder is recommended before transplantation. However, an abnormal urinary bladder is no longer a contraindication to renal transplantation $[21,28,31]$.

3. Miscellaneous

Other measures include high oral fluid intake and hygienic measures, especially in the adolescent, sexually active patients. Sometimes, home testing of urine by the parent or patient with dipsticks is helpful to detect UTI early. No data are available for alternative methods, such as cranberry juice, urine acidification, etc.

\section{Summary and conclusion}

Children after renal transplantation are at high risk for UTIs. Although anatomical factors including neurogenic bladder increase the risk for UTI, the high prevalence in girls and in patients with nonanatomical underlying disorders indicate that further risk factors are present. The severe renal dysfunction during fUTI and inflammatory response indicates that fUTI has to be regarded as a serious complication, endangering long-term graft survival. There- 
fore, prophylactic measures including antibiotic prophylaxis and bladder training should be considered. Prospective studies are urgently needed to evaluate precise mechanisms and the value of preventive measures of this important complication.

\section{Questions}

(Answers appear following the reference list)

Which answer is true: UTIs after KTX

1. Pseudomonas and Enterococcus spp. cause UTIs after renal transplantation:

a. In the same frequency as in unimmunocompromised patients

b. More frequently than in immunologically healthy children

c. Less frequently than in immunologically healthy children

d. Are specifically related to graft rejection episodes

e. None of the above is true

2. Which answer is not true: diagnostic workup of a patient after renal transplantation with fever typically includes:
a. Renal biopsy
b. Renal ultrasound, including Doppler ultrasonography
c. Urine culture
d. Laboratory testing, including full blood count, serum creatinine and CRP
e. Clinical examination

3. Long-term complications after fUTI in patients after KTX:
a. Are irrelevant
b. Develop only in patients with acquired diseases
c. Have been documented and include accelerated graft loss and chronic allograft nephropathy
d. Are only present in females
e. Can be prevented by specific immunosuppression

4. Which answer is not true: the main differential diagnosis of a patient with kidney graft dysfunction and fever includes:
a. Cytomegalovirus infection
b. Acute rejection
c. Transplant pyelonephritis
d. Dehydration during viral illness
e. Malnutrition

5. Risk factors for UTI after KTX include:
a. Underlying urinary tract pathology
b. Female gender
c. VUR into kidney transplant
d. Voiding dysfunction and constipation
e. All of the above

\section{References}

1. Offner G, Latta K, Hoyer PF, Baum HJ, Ehrich JH, Pichlmayr R, Brodehl J (1999) Kidney transplanted children come of age. Kidney Int 55:1509-1517

2. Filler G, Webb NJ, Milford DV, Watson AR, Gellermann J, Tyden G, Grenda R, Vondrak K, Hughes D, Offner G, Griebel M, Brekke IB, McGraw M, Balzar E, Friman S, Trompeter R (2005) Four-year data after pediatric renal transplantation: a randomized trial of tacrolimus vs. cyclosporin microemulsion. Pediatr Transplant 9:498-503

3. Buscher R, Vester U, Wingen AM, Hoyer PF (2004) Pathomechanisms and the diagnosis of arterial hypertension in pediatric renal allograft recipients. Pediatr Nephrol 19:1202-1211

4. Giral M, Pascuariello G, Karam G, Hourmant M, Cantarovich D, Dantal J, Blancho G, Coupel S, Josien R, Daguin P, Mechineau S, Soulillou JP (2002) Acute graft pyelonephritis and long-term kidney allograft outcome. Kidney Int 61:1880-1886

5. Cairns HS, Spencer S, Hilson AJ, Rudge CJ, Neild GH (1994) 99mTc-DMSA imaging with tomography in renal transplant recipients with abnormal lower urinary tracts. Nephrol Dial Transplant 9:1157-1161

6. Mueller T, Resinger C, Ruffingshofer D, Arbeiter K, Balzar E, Aufricht $C$ (2003) Urinary tract infections beyond the early posttransplant period in pediatric renal graft recipients. Wien Klin Wochenschr 115:385-388

7. Howie AJ, Buist LJ, Coulthard MG (2002) Reflux nephropathy in transplants. Pediatr Nephrol 17:485-490

8. Chuang P, Parikh CR, Langone A (2005) Urinary tract infections after renal transplantation: a retrospective review at two US transplant centers. Clin Transplant 19:230-235

9. Ranchin B, Chapuis F, Dawhara M, Canterino I, Hadj-Aissa A, Said MH, Parchoux B, Dubourg L, Pouillaude JM, Floret D, Martin X, Cochat P (2000) Vesicoureteral reflux after kidney transplantation in children. Nephrol Dial Transplant 15:1852-1858

10. Dunn SP, Vinocur CD, Hanevold C, Wagner CW, Weintraub WH (1987) Pyelonephritis following pediatric renal transplant: increased incidence with vesicoureteral reflux. J Pediatr Surg 22: 1095-1099

11. Hansson S, Jodal U (1999) Urinary tract infection. In: Barrat TM, Avner E, Harmon WE (eds) Pediatric nephrology. 4th edn. Baltimore, Lippincott Williams \& Wilkins, pp. 835-850

12. American Academy of Pediatrics (1999) Practice parameter: the diagnosis, treatment, and evaluation of the initial UTI in febrile infants and young children. American Academy of Pediatrics. Committee on Quality Improvement. Subcommittee on Urinary Tract Infection. Pediatrics 103:843-852

13. Dyck RF (1988) C-reactive protein: a better indicator of complications other than acute rejection in renal allograft recipients? Clin Biochem 21:359-362

14. John U, Everding AS, Kuwertz-Broking E, Bulla M, MullerWiefel DE, Misselwitz J, Kemper MJ (2006) High prevalence of febrile urinary tract infections after paediatric renal transplantation. Nephrol Dial Transplant 21:3269-3274

15. Coulthard MG, Keir MJ (2006) Reflux nephropathy in kidney transplants, demonstrated by dimercaptosuccinic acid scanning. Transplantation 82:205-210

16. Hutchinson C, Beckett M, Kiratli P, Gordon I, Trompeter RS, Rees L (2003) The significance of a defect on DMSA scan in children with renal transplants. Pediatr Transplant 7:441-445

17. Neuhaus TJ, Schwobel M, Schlumpf R, Offner G, Leumann E, Willi U (1997) Pyelonephritis and vesicoureteral reflux after renal transplantation in young children. J Urol 157:1400-1403

18. Audard V, Amor M, Desvaux D, Pastural M, Baron C, Philippe R, Pardon A, Dahmane D, Lang P, Grimbert P (2005) Acute graft pyelonephritis: a potential cause of acute rejection in renal transplant. Transplantation 80:1128-1130 
19. Eilers E, Moter A, Bollmann R, Haffner D, Querfeld U (2007) Intrarenal abscesses due to Ureaplasma urealyticum in a transplanted kidney. J Clin Microbiol 45:1066-1068

20. Maraha B, Bonten $H$, van Hooff $H$, Fiolet $H$, Buiting AG, Stobberingh EE (2001) Infectious complications and antibiotic use in renal transplant recipients during a 1-year follow-up. Clin Microbiol Infect 7:619-625

21. Koo HP, Bunchman TE, Flynn JT, Punch JD, Schwartz AC, Bloom DA (1999) Renal transplantation in children with severe lower urinary tract dysfunction. J Urol 161:240-245

22. Pelle G, Vimont S, Levy PP, Hertig A, Ouali N, Chassin C, Arlet G, Rondeau E, Vandewalle A (2007) Acute pyelonephritis represents a risk factor impairing long-term kidney graft function. Am J Transplant 7:899-907

23. Van der Weide MJ, Cornelissen EA, Van Achterberg T, Smits JP, Feitz WF (2006) Dysfunction of lower urinary tract in renal transplant children with nephrologic disease. Urology 67:1060-1065

24. Sharifian M, Rees L, Trompeter RS (1998) High incidence of bacteriuria following renal transplantation in children. Nephrol Dial Transplant 13:432-435

25. Ergin F, Arslan H, Yapar G, Karakayali H, Haberal M (2003) Urinary tract infections in renal transplant recipients. Transplant Proc 35:2685-2686

26. Jodal U, Winberg J (1987) Management of children with unobstructed urinary tract infection. Pediatr Nephrol 1:647-656

27. Benfield MR, McDonald RA, Bartosh S, Ho PL, Harmon W (2003) Changing trends in pediatric transplantation: 2001 Annual Report of the North American Pediatric Renal Transplant Cooperative Study. Pediatr Transplant 7:321-335

28. Adams J, Mehls O, Wiesel M (2004) Pediatric renal transplantation and the dysfunctional bladder. Transpl Int 17:596-602

29. Mochon M, Kaiser BA, Dunn S, Palmer J, Polinsky MS, Schulman SL, Flynn JT, Baluarte HJ (1992) Urinary tract infections in children with posterior urethral valves after kidney transplantation. J Urol 148:1874-1876

30. Capizzi A, Zanon GF, Zacchello G, Rigamonti W (2004) Kidney transplantation in children with reconstructed bladder. Transplantation 77:1113-1116

31. Neild GH, Dakmish A, Wood S, Nauth-Misir R, Woodhouse CR (2004) Renal transplantation in adults with abnormal bladders. Transplantation 77:1123-1127

32. Saedi NA, Schulman SL (2003) Natural history of voiding dysfunction. Pediatr Nephrol 18:894-897

33. Herthelius M, Oborn H (2006) Bladder dysfunction in children and adolescents after renal transplantation. Pediatr Nephrol 21:725-728

34. Herthelius M, Oborn $\mathrm{H}$ (2007) Urinary tract infections and bladder dysfunction after renal transplantation in children. J Urol 177:1883-1886

35. Casale P, Grady RW, Mitchell ME, Healey P (2005) Recurrent urinary tract infection in the post-transplant reflux nephropathy patient: is reflux in the native ureter the culprit? Pediatr Transplant 9:324-327

36. Basiri A, Otookesh H, Simforoosh N, Hosseini R, HosseiniMoghaddam SM, Sharifian M (2006) Does pre-transplantation antireflux surgery eliminate post-renal transplantation pyelonephritis in children? J Urol 175:1490-1492

37. Kamath NS, John GT, Neelakantan N, Kirubakaran MG, Jacob CK (2006) Acute graft pyelonephritis following renal transplantation. Transpl Infect Dis 8:140-147
38. Abbott KC, Swanson SJ, Richter ER, Bohen EM, Agodoa LY, Peters TG, Barbour G, Lipnick R, Cruess DF (2004) Late urinary tract infection after renal transplantation in the United States. Am J Kidney Dis 44:353-362

39. Sadeghi M, Daniel V, Naujokat C, Wiesel M, Hergesell O, Opelz G (2005) Strong inflammatory cytokine response in male and strong anti-inflammatory response in female kidney transplant recipients with urinary tract infection. Transpl Int 18:177-185

40. Luke PP, Herz DB, Bellinger MF, Chakrabarti P, Vivas CA, Scantlebury VP, Hakala TR, Jevnikar AM, Jain A, Shapiro R, Jordan ML (2003) Long-term results of pediatric renal transplantation into a dysfunctional lower urinary tract. Transplantation 76:1578-1582

41. Fontana I, Ginevri F, Arcuri V, Basile G, Nocera A, Beatini M, Bonato L, Barocci S, Bertocchi M, Manolitsi O, Valente R, Draghi P, Gusmano R, Valente U (1999) Vesico-ureteral reflux in pediatric kidney transplants: clinical relevance to graft and patient outcome. Pediatr Transplant 3:206-209

42. Rice JC, Peng T, Kuo YF, Pendyala S, Simmons L, Boughton J, Ishihara K, Nowicki S, Nowicki BJ (2006) Renal allograft injury is associated with urinary tract infection caused by Escherichia coli bearing adherence factors. Am J Transplant 6:2375-2383

43. Prat C, Dominguez J, Rodrigo C, Gimenez M, Azuara M, Jimenez O, Gali N, Ausina V (2003) Elevated serum procalcitonin values correlate with renal scarring in children with urinary tract infection. Pediatr Infect Dis J 22:438-442

44. Wangoo A, Laban C, Cook HT, Glenville B, Shaw RJ (1997) Interleukin-10- and corticosteroid-induced reduction in type I procollagen in a human ex vivo scar culture. Int J Exp Pathol 78: 33-41

45. Benador D, Neuhaus TJ, Papazyan JP, Willi UV, Engel-Bicik I, Nadal D, Slosman D, Mermillod B, Girardin E (2001) Randomised controlled trial of three day versus 10-day intravenous antibiotics in acute pyelonephritis: effect on renal scarring. Arch Dis Child 84:241-246

46. Haller M, Brandis M, Berner R (2004) Antibiotic resistance of urinary tract pathogens and rationale for empirical intravenous therapy. Pediatr Nephrol 19:982-986

47. Valera B, Gentil MA, Cabello V, Fijo J, Cordero E, Cisneros JM (2006) Epidemiology of urinary infections in renal transplant recipients. Transplant Proc 38:2414-2415

48. Alangaden GJ, Thyagarajan R, Gruber SA, Morawski K, Garnick J, El-Amm JM, West MS, Sillix DH, Chandrasekar PH, Haririan A (2006) Infectious complications after kidney transplantation: current epidemiology and associated risk factors. Clin Transplant 20:401-409

49. Pape L, Gunzer F, Ziesing S, Pape A, Offner G, Ehrich JH (2004) Bacterial pathogens, resistance patterns and treatment options in community acquired pediatric urinary tract infection. Klin Padiatr 216:83-86

\section{Answers}

1. b
2. a
3. c
4. e
5. e 